\title{
Higher Power Residue Codes and the Leech Lattice
}

\author{
MEHRDAD AHMADZADEH RAJI \\ Department of Mathematical Sciences, University of Exeter, Exeter EX4 4QE, UK
}

m.raji@ex.ac.uk

Received August 30, 2001; Revised January 5, 2004; Accepted January 27, 2004

\begin{abstract}
We shall consider higher power residue codes over the ring $\mathbf{Z}_{4}$. We will briefly introduce these codes over $\mathbf{Z}_{4}$ and then we will find a new construction for the Leech lattice. A similar construction is used to construct some of the other lattices of rank 24.
\end{abstract}

Keywords: self-dual code, even unimodular lattice, Hensel lifting

\section{Introduction}

Let $p, l$ be prime numbers. Let $\mathbf{F}_{l}$ be a finite field of order $l$ and $\mathbf{F}_{l^{2}}$ be a finite extension of degree 2 over the finite field $\mathbf{F}_{l}$. Chapman introduced higher power residue codes $\mathrm{W}$ over the Galois field $\mathbf{F}_{l^{2}}$ [3]. These are codes over $\mathbf{F}_{l^{2}}$ but linear only over $\mathbf{F}_{l}$, not $\mathbf{F}_{l^{2}}$, and they satisfy $W \otimes_{\mathbf{F}_{l}} \mathbf{F}_{l^{2}}=\left(\mathbf{F}_{l^{2}}\right)^{p+1}$. They depend on characters $\chi: \mathbf{F}_{p}^{*} \rightarrow \mathbf{F}_{l^{2}}^{*}$ where for a given field $\mathbf{F}, \mathbf{F}^{*}=\mathbf{F}-\{0\}$.

Here we begin the task of generalizing this construction to Galois rings. We confine ourselves here to the Galois ring $\mathbf{Z}_{4}[\omega]$ where $\omega^{2}+\omega+1=0$. The characters we consider here have orders 3 or 6 , so we call the corresponding codes cubic or sextic residue codes.

When $p \equiv 7(\bmod 24)$ we can combine sextic residue codes over $\mathbf{Z}_{4}[\omega]$ with quadratic residue codes to form self-dual codes of length $3(p+1)$ over $\mathbf{Z}_{4}$. These yield unimodular lattices. The first case is $p=7$ which we deal with in detail.

\section{Higher power residue codes over $\mathbf{Z}_{4}$}

Let $\mathbf{Z}_{4}[\omega]=\left\{a+b \omega: a, b \in \mathbf{Z}_{4}\right\}$ where $\omega$ is a primitive cube root of unity. So $\omega$ satisfies $\omega^{2}+\omega+1=0$. We define the following automorphism on $\mathbf{Z}_{4}[\omega]$.

$$
\begin{aligned}
{ }^{-}: \mathbf{Z}_{4}[\omega] & \longrightarrow \mathbf{Z}_{4}[\omega] \\
a+b \omega & \longmapsto a+b \omega^{2}
\end{aligned}
$$

This is an automorphism of order two.

Let $p$ be a prime number with $p \equiv 1(\bmod 6)$. Now consider

$$
\Omega=\left\{(\alpha, \beta): \alpha, \beta \in \mathbf{Z}_{p}\right\}-\{(0,0)\}
$$


Define $\mathbf{Z}_{4}[\omega]^{*}=\mathbf{Z}_{4}[\omega]-\left\{0,2,2 \omega, 2 \omega^{2}\right\}$ the group of units of $\mathbf{Z}_{4}[\omega]$. Let $\chi: \mathbf{Z}_{p}^{*} \rightarrow \mathbf{Z}_{4}[\omega]^{*}$ be a character of order 3 or 6 such that $\chi(\alpha) \in\left\{ \pm \omega^{j}\right\}$ where $j \in\{0,1,2\}, \alpha \in \mathbf{Z}_{p}$ and $\chi(\alpha)^{-1}=\overline{\chi(\alpha)}$. We define the $\mathbf{Z}_{4}[\omega]$-module $M$ with generators $e_{v}$ where $v \in \Omega$ and relations $e_{\alpha v}=\chi(\alpha) e_{v}$. Let

$$
\Delta=\left\{e_{\infty}, e_{0}, e_{1}, \ldots, e_{p-1}\right\}
$$

where $e_{\infty}=e_{(1,0)}$ and $e_{\alpha}=e_{(\alpha, 1)}$ for $\alpha \in \mathbf{Z}_{p}$. We suppose that there is no non-trivial linear relation among the elements of $\Delta$. So the $\mathbf{Z}_{4}[\omega]$-module $M$ can be generated as a finitely generated module of rank $p+1$ by linear combinations of the elements of $\Delta$.

We denote the general linear group of degree 2 over $\mathbf{Z}_{p}$ by $\mathbf{G L}(2, p)$. Define the action of $\mathbf{G L}(2, p)$ on the projective line $\mathbf{P}^{1}\left(\mathbf{Z}_{p}\right)$ over $\mathbf{Z}_{p}$ as

$$
v \cdot\left(\begin{array}{ll}
\alpha & \beta \\
\gamma & \delta
\end{array}\right)=\frac{v \alpha+\gamma}{v \beta+\delta}
$$

and

$$
\infty \cdot\left(\begin{array}{ll}
\alpha & \beta \\
\gamma & \delta
\end{array}\right)=\frac{\alpha}{\beta} .
$$

Therefore if $A=\left(\begin{array}{ll}\alpha & \beta \\ \gamma & \delta\end{array}\right)$, we have

$$
e_{v} A=\chi(v \beta+\delta) e_{v \cdot A}
$$

for $v \neq \infty$ and

$$
e_{\infty} A=\chi(\beta) e_{\infty \cdot A}
$$

One can easily verify that

$$
e_{\alpha v} A=e_{\alpha v A}=\chi(\alpha) e_{v A}=\chi(\alpha) e_{v} A .
$$

Definition 2.1 Let $f: M_{1} \rightarrow M_{2}$ be a map between $\mathbf{Z}_{4}[\omega]$-modules $M_{1}$ and $M_{2}$. We say $f$ is semi-linear if $f(\lambda m)=\bar{\lambda} f(m)$ for all $\lambda \in \mathbf{Z}_{4}[\omega], m \in M$.

We aim to find a $\mathbf{Z}_{4}$-submodule $W$ of $M$ with the property that the natural map $W \otimes \mathbf{Z}_{4}$ $\mathbf{Z}_{4}[\omega] \rightarrow M$ is an isomorphism. This is equivalent to $M=W \oplus \omega W$.

Lemma 2.1 Let $W$ be a $\mathbf{Z}_{4}$-submodule of $M$ with $M=W \oplus \omega W$. Then

$$
\begin{array}{ccc}
\tau: M & \rightarrow \quad M \\
r+\omega s & \mapsto r+\bar{\omega} s
\end{array}
$$

where $r, s \in W$, is a semi-linear involution. 
Proof: Let $r, s \in W$ and $\lambda \in \mathbf{Z}_{4}[\omega]$. Thus $\lambda=a+b \omega$ for some $a, b \in \mathbf{Z}_{4}$. Then

$$
\begin{aligned}
\tau(\lambda(r+\omega s)) & =\tau(a r+b r \omega+a s \omega+b s(-1-\omega)) \\
& =a r-b s+(b r+a s-b s) \bar{\omega} \\
& =\bar{\lambda}(r+\bar{\omega} s) \\
& =\bar{\lambda}(\tau(r+\omega s)) .
\end{aligned}
$$

Hence $\tau$ is semi-linear. Finally

$$
\tau^{2}(r+\omega s)=\tau(r+\bar{\omega} s)=r+\omega s
$$

The converse of Lemma 2.1 is also true.

Lemma 2.2 Let $\tau: M \rightarrow M$ be a semi-linear involution and $W=\{m \in M: \tau(m)=m\}$. Then $W$ is a $\mathbf{Z}_{4}$-submodule and $M=W \oplus \omega W$.

Proof: Suppose that $\tau: M \rightarrow M$ is a semi-linear involution. Since $\bar{a}=a$ for all $a \in \mathbf{Z}_{4}$, the set $W$ is a $\mathbf{Z}_{4}$-submodule of $M$. Since $\omega \neq \omega^{2}, \lambda=\omega-\bar{\omega} \in \mathbf{Z}_{4}[\omega]^{*}$.

It is clear that $\bar{\lambda}=-\lambda$. We shall show that for all $m \in M$ there exist $r, s \in W$ such that $m=r+\omega s$. For this set

$$
\begin{aligned}
r & =\frac{1}{\lambda}(\omega \tau(m)-\bar{\omega} m) \\
s & =\frac{1}{\lambda}(m-\tau(m)) .
\end{aligned}
$$

Therefore

$$
\tau(r)=\frac{\bar{\omega}}{\bar{\lambda}} m-\frac{1}{\bar{\lambda}} \omega \tau(m)=\frac{-1}{\lambda}(\bar{\omega} m-\omega \tau(m))=r
$$

and

$$
\tau(s)=s .
$$

So we have proved that $M=W+\omega W$. Now suppose $\hat{r} \in W \cap \omega W$. Since $\hat{r} \in W$, we have $\tau(\hat{r})=\hat{r}$. On the other hand, $\hat{r} \in \omega W$ so $\hat{r}=\omega \hat{s}$ for some $\hat{s} \in W$. Hence $\hat{s}=\omega^{-1} \hat{r}$ and

$$
\tau(\hat{r})=\tau(\omega \hat{s})=\bar{\omega} \hat{s}=\bar{\omega} \omega^{-1} \hat{r}=\omega^{2} \omega^{-1} \hat{r}=\omega \hat{r}
$$

Since $1-\omega \in \mathbf{Z}_{4}[\omega]^{*}$, we have $\hat{r}=0$. Hence

$$
M=W \oplus \omega W .
$$


Denote by $\mathbf{S L}(2, p)$ the set of all $2 \times 2$ matrices $A$ with entries in $\mathbf{Z}_{p}$ such that $\operatorname{det} A=1$. Then $\mathbf{S L}(2, p)$ is called the 2-dimensional special linear group over $\mathbf{Z}_{p}$. We aim to find such a $W$ invariant under the action of $\mathbf{S L}(2, p)$.

Lemma 2.3 Let $\tau: M \rightarrow M$ be a semi-linearinvolution, and $W=\{m \in M: m=\tau(m)\}$. Then $W$ is invariant under the action of $\mathbf{S L}(2, p)$ if and only if $\tau(m A)=\tau(m) A$ for all $A \in \mathbf{S L}(2, p)$ and $m \in M$.

Proof: Suppose $W$ is invariant under $\mathbf{S L}(2, p)$ and $r, s \in W$. Then

$$
\begin{aligned}
\tau((r+\omega s) A) & =\tau(r A+\omega s A)=\tau(r A)+\tau(\omega s A) \\
& =r A+\bar{\omega} s A=(r+\bar{\omega} s) A \\
& =\tau(r+\omega s) A .
\end{aligned}
$$

Conversely if $\tau(m A)=\tau(m) A$ for all $m \in M$ and $A \in \mathbf{S L}(2, p)$, then for $m \in W$

$$
\tau(m A)=\tau(m) A=m A \text {. }
$$

Hence $W$ is invariant under $\mathbf{S L}(2, p)$.

Quaternary quadratic residue codes are invariant under the corresponding action of $\mathbf{S L}(2, p)$ defined from the quadratic character $\chi(a)=\left(\frac{a}{p}\right)$ (see [2]).

Definition 2.2 We consider such a submodule $W$ which is invariant under the action of $\operatorname{SL}(2, p)$ according to Lemma 2.3. We call $W$ a higher power residue code. In particular when $\chi$ is a character of order 3 we call $W$ a cubic residue code and when $\chi$ has order 6 we call $W$ a sextic residue code.

Now we are going to define a hermitian structure on $M$.

Definition 2.3 A $\mathbf{Z}_{4}$ - bilinear form $\Phi: M \times M \rightarrow \mathbf{Z}_{4}[\omega]$ satisfying

(1) $\Phi\left(\lambda_{1} m_{1}+\lambda_{2} m_{2}, \hat{m}_{1}\right)=\bar{\lambda}_{1} \Phi\left(m_{1}, \hat{m}_{1}\right)+\bar{\lambda}_{2} \Phi\left(m_{2}, \hat{m}_{1}\right)$

(2) $\Phi\left(m_{1}, \lambda_{1} \hat{m}_{1}+\lambda_{2} \hat{m}_{2}\right)=\lambda_{1} \Phi\left(m_{1}, \hat{m}_{1}\right)+\lambda_{2} \Phi\left(m_{1}, \hat{m}_{2}\right)$

for all $m_{1}, m_{2}, \hat{m}_{1}, \hat{m}_{2} \in M, \lambda_{1}, \lambda_{2} \in \mathbf{Z}_{4}[\omega]$ is called a sesquilinear form on $\mathbf{M}$.

Lemma 2.4 Define a sesquilinear form $\Phi$ on $M$ by

$$
\Phi\left(e_{\alpha}, e_{\beta}\right)= \begin{cases}1 & \alpha=\beta \\ 0 & \alpha \neq \beta\end{cases}
$$

for $\alpha, \beta \in \mathbf{P}^{1}\left(\mathbf{Z}_{p}\right)$. Then $\Phi$ has the following properties:

(i) $\Phi\left(e_{v}, e_{w}\right)=\overline{\Phi\left(e_{w}, e_{v}\right)}$ for all $v, w \in \Omega$

(ii) $\Phi\left(e_{v} A, e_{w} A\right)=\Phi\left(e_{v}, e_{w}\right)$ for all $v, w \in \Omega$ 


\section{Proof:}

(i) First of all suppose $v$ and $w$ are linearly independent. In this case both sides of (i) are zero. Now suppose $w=\alpha v$. Therefore:

$$
\overline{\Phi\left(e_{w}, e_{v}\right)}=\overline{\chi(\alpha)}
$$

On the other hand $v=\alpha^{-1} w$ and

$$
\Phi\left(e_{w}, e_{v}\right)=\chi\left(\alpha^{-1}\right)
$$

The result follows from (4), (5) and the fact that $\chi\left(\alpha^{-1}\right)=\overline{\chi(\alpha)}$.

(ii) One can achieve the result by using the same process as (i) and definition of $e_{v} A$ and applying (2).

Corollary 2.1 Let $\Phi$ be as in Lemma 2.4. Then for all $m_{1}, m_{2} \in M$ and $A \in \mathbf{S L}(2, p)$ we have

(i) $\Phi\left(m_{2}, m_{1}\right)=\overline{\Phi\left(m_{1}, m_{2}\right)}$

(ii) $\Phi\left(m_{1} A, m_{2} A\right)=\Phi\left(m_{1}, m_{2}\right)$.

\section{Proof:}

(i) is clear by sesquilinearity of $\Phi$.

(ii) follows from (ii) of Lemma 2.4 and the linearity of $\Phi$.

Recall $\Omega=\left(\mathbf{Z}_{p} \times \mathbf{Z}_{p}\right)-\{(0,0)\}$

Lemma 2.5 Let $\tau$ be a semi-linear involution $\tau: M \rightarrow M$ such that $\tau(m A)=\tau(m) A$ for all $A \in \mathbf{S L}(2, p)$ and $m \in M$, and $\chi: \mathbf{Z}_{p}^{*} \rightarrow \mathbf{Z}_{4}[\omega]^{*}$ be a character of order $s>2$. Define $\Psi: \Omega \times \Omega \rightarrow \mathbf{Z}_{4}[\omega]^{*}$ by $\Psi(v, w)=\Phi\left(\tau\left(e_{v}\right),\left(e_{w}\right)\right)$. Then $\Psi$ satisfies the following.

(i) $\Psi(\alpha v, \beta w)=\chi(\alpha \beta) \Psi(v, w)$ for $\alpha, \beta \in \mathbf{Z}_{p}$

(ii) $\Psi(v A, w A)=\Psi(v, w)$

(iii) $\Psi(v, w)=0 \quad$ whenever $v$ and $w$ are linearly dependent.

\section{Proof:}

(i)

$$
\begin{aligned}
\Psi(\alpha v, \beta w) & =\Phi\left(\tau\left(e_{\alpha v}\right), e_{\beta w}\right)=\Phi\left(\tau\left(\chi(\alpha) e_{v}\right), \chi(\beta) e_{w}\right) \\
& =\Phi\left(\overline{\chi(\alpha)} \tau\left(e_{v}\right), \chi(\beta) e_{w}\right)=\chi(\alpha) \chi(\beta) \Phi\left(\tau\left(e_{v}\right), e_{w}\right) \\
& =\chi(\alpha \beta) \Psi(v, w) .
\end{aligned}
$$


(ii)

$$
\begin{aligned}
\Psi(v A, w A) & =\Phi\left(\tau\left(e_{v A}\right), e_{w A}\right)=\Phi\left(\tau\left(e_{v} A\right), e_{w} A\right) \\
& =\Phi\left(\tau\left(e_{v}\right) A, e_{w} A\right) \\
& =\Phi\left(\tau\left(e_{v}\right), e_{w}\right) \\
& =\Psi(v, w)
\end{aligned}
$$

(iii) Suppose $\alpha$ is an element of $\mathbf{Z}_{p}^{*}$ such that $\chi(\alpha) \neq \pm 1$. Such an element exists, since otherwise, the order of $\chi$ does not exceed two. Now we can find a matrix $A \in \mathbf{S L}(2, p)$ such that $v A=\alpha v$, and then also $w A=\alpha w$. Therefore, $\Psi(v, w)=\Psi(v A, w A)=$ $\Psi(\alpha v, \alpha w)=\chi(\alpha)^{2} \Psi(v, w)$. Hence, $\Psi(v, w)=0$.

Lemma 2.6 Let $\Psi$ be as in Lemma 2.5 and $v=(\alpha, \beta), w=(\gamma, \delta)$ be linearly independent elements of $\Omega, A=\left(\begin{array}{ll}\alpha & \beta \\ \gamma & \delta\end{array}\right)$ and $\hat{x}=\operatorname{det} A$.

(i) There exists some $\zeta \in \mathbf{Z}_{4}[\omega]$ such that $\Psi(v, w)=\zeta \chi(\hat{x})$

(ii) $\Psi(w, v)=\chi(-1) \Psi(v, w)$.

\section{Proof:}

(i) Let $v_{0}=(1,0), w_{0}=(0,1)$. Hence $v=v_{0} A^{\prime}, w=\hat{x} w_{0} A^{\prime}$ where

$$
A^{\prime}=\left(\begin{array}{cc}
\hat{x}^{-1} \gamma & \hat{x}^{\underline{\beta}} \delta
\end{array}\right) \in \mathbf{S L}(2, p)
$$

Then

$$
\Psi(v, w)=\Psi\left(v_{0} A^{\prime}, \hat{x} w_{0} A^{\prime}\right)=\chi(\hat{x}) \Psi\left(v_{0}, w_{0}\right) .
$$

Taking $\zeta$ as $\Psi\left(v_{0}, w_{0}\right)$, completes the proof.

(ii) $\Psi(w, v)=\zeta \chi(-\hat{x})=\zeta \chi(-1) \chi(\hat{x})=\chi(-1) \Psi(v, w)$.

Proposition 2.1 Let $\zeta$ be an element of $\mathbf{Z}_{4}[\omega]$ satisfying the conditions of Lemma 2.6 and $\tau: M \rightarrow M$ be a semi-linear involution which satisfies $\tau(m A)=\tau(m) A$, for all $A \in \mathbf{S L}(2, p)$ and $m \in M$. Then

$$
\tau\left(e_{v}\right)=\sum_{w \in \mathbf{P}^{1}\left(\mathbf{Z}_{p}\right)} \overline{\zeta U_{v w}} e_{w}
$$

where

$$
U_{\alpha \beta}= \begin{cases}\chi(\alpha-\beta) & \text { if } \beta \neq \infty, \alpha \neq \infty \\ \chi(-1) & \text { if } \beta=\infty, \alpha \neq \infty \\ 1 & \text { if } \alpha=\infty, \beta \neq \infty \\ 0 & \text { if } \alpha=\infty, \beta=\infty\end{cases}
$$


and $\zeta$ satisfies the equation

$$
p \chi(-1) \zeta \bar{\zeta}=1
$$

Proof: Using Lemmas 2.5 and 2.6 shows that

$$
\begin{aligned}
\tau\left(e_{v}\right) & =\sum_{w \in \mathbf{P}^{1}\left(\mathbf{Z}_{p}\right)} \Phi\left(e_{w}, \tau\left(e_{v}\right)\right) e_{w}=\sum_{w \in \mathbf{P}^{1}\left(\mathbf{Z}_{p}\right)} \overline{\Phi\left(\tau\left(e_{v}\right), e_{w}\right)} e_{w} \\
& =\sum_{w \in \mathbf{P}^{1}\left(\mathbf{Z}_{p}\right)} \overline{\Psi(v, w)} e_{w}=\sum_{w \in \mathbf{P}^{1}\left(\mathbf{Z}_{p}\right)} \overline{\zeta U_{v w}} e_{w}
\end{aligned}
$$

where $U$ is the matrix defined by (10). Therefore,

$$
\tau\left(e_{\infty}\right)=\bar{\zeta} \sum_{i=0}^{p-1} e_{i}
$$

and so

$$
\begin{aligned}
1 & =\Phi\left(e_{\infty}, e_{\infty}\right)=\Phi\left(\tau\left(e_{\infty}\right)^{2}, e_{\infty}\right) \\
& =\Phi\left(\tau\left(\tau\left(e_{\infty}\right)\right), e_{\infty}\right)=\Phi\left(\tau\left(\bar{\zeta} \sum_{i=0}^{p-1} e_{i}\right), e_{\infty}\right) \\
& =\Phi\left(\sum_{i=0}^{p-1} \zeta \tau\left(e_{i}\right), e_{\infty}\right)=\bar{\zeta}\left(\sum_{i=0}^{p-1} \Phi\left(\tau\left(e_{i}\right), e_{\infty}\right)\right. \\
& =\bar{\zeta} \zeta p \chi(-1) .
\end{aligned}
$$

Therefore

$$
p \chi(-1) \zeta \bar{\zeta}=1
$$

Remark 2.1 If $\zeta$ satisfies (12) and $\tau$ is defined by (9) then by Lemma 2.4,

$$
\Phi\left(e_{\alpha}, e_{\beta}\right)=\Phi\left(\tau^{2}\left(e_{\alpha}\right), e_{\beta}\right)
$$

Therefore, $\tau^{2}(m)=m$ for all $m \in M$. This shows that $\tau$ is a semi-linear involution. Moreover,

$$
\begin{aligned}
\Phi\left(\tau\left(e_{v} A\right), w\right) & =\Phi\left(\tau\left(e_{v A}\right), w\right)=\Psi(v A, w)=\Psi\left(v, w A^{-1}\right) \\
& =\Phi\left(\tau\left(e_{v}\right), w A^{-1}\right)=\Phi\left(\tau\left(e_{v}\right) A, w\right) .
\end{aligned}
$$

Hence $\tau(m A)=\tau(m) A$, for all $m \in M$. So by Lemma 2.3, $W=\{m \in M: \tau(m)=m\}$ is invariant under the action of $\mathbf{S L}(2, p)$. 
Remark 2.1 shows the existence of a semi-linear involution $\tau$ such that $W$ is invariant under the action of $\mathbf{S L}(2, p)$. In fact any semi-linear involution $\tau$ on $M$ gives a Higher power residue code $W$ and semi-linear involutions are correspond to the solutions of (12) via (9).

One of the prominent questions is : how many different higher power residue codes are there in each case. We need to prove the following lemma

Lemma 2.7 Let $W$ be a higher power residue code over $\mathbf{Z}_{4}[\omega]$ then $\lambda W$ for $\lambda \in \mathbf{Z}_{4}[\omega]^{*}$ is a higher power residue code.

Proof: What we shall do is to find a semi-linear involution $\tau$ such that $\hat{\tau}(\lambda W)=\lambda W$. Let $\hat{\tau}=\varepsilon \tau$. So we have

$$
\dot{\tau}(\lambda m)=\varepsilon \tau(\lambda m)=\varepsilon \bar{\lambda} \tau(m)=\varepsilon \bar{\lambda} m
$$

for $m \in W$. So it suffices to choose $\varepsilon=\lambda / \bar{\lambda}$. Obviously $\varepsilon \bar{\varepsilon}=1$ and this proves that $\hat{\tau}$ is a semi-linear involution.

Proposition 2.2 The higher power residue code is unique up to multiplication by an element of $\mathbf{Z}_{4}[\omega]^{*}$.

Proof: Let $W$ and $W$ be higher power residue codes. Then they are respectively the fixed sets of semi-linear involutions $\tau$ and $\tau$, and they are invariant under the action of $\mathbf{S L}(2, p)$. By (9) and (12) $\bar{\tau}=\epsilon \tau$ where $\epsilon \bar{\epsilon}=1$. Such an $\epsilon$ has the form $\lambda / \bar{\lambda}$ and so $W=\lambda W$ by the argument of Lemma 2.7.

Proposition 2.3 Let $\tau$ be the unique semi-linear involution $\tau: M \rightarrow M$ which is defined by (9) and $S$ be the set of $\eta \in \mathbf{Z}_{4}[\omega]^{*}$ such that $\eta+\bar{\eta} \in \mathbf{Z}_{4}[\omega]^{*}$. Define

$$
\begin{aligned}
h: M & \rightarrow M \\
m & \mapsto \eta m+\bar{\eta} \tau(m)
\end{aligned}
$$

for some $\eta \in S$. Then $W$ is the image of $h$.

Proof: If $m \in W$ and $\eta \in S$ then $\eta m+\tau(\eta m)=(\eta+\bar{\eta}) m$. Set $n=(\eta+\bar{\eta})^{-1} m$. Therefore $h(n)=m$.

Definition 2.4 Define a $\boldsymbol{Z}_{4}$-bilinear map

$$
\begin{aligned}
{[\quad, \quad]: M \times M } & \rightarrow Z_{4} \\
\left(m_{1}, m_{2}\right) & \longmapsto \Phi\left(m_{1}, m_{2}\right)+\overline{\Phi\left(m_{1}, m_{2}\right)} .
\end{aligned}
$$


We denote the dual space of $W$ by

$$
W^{\prime}=\left\{m_{2} \in M:\left[m_{1}, m_{2}\right]=0 \quad \text { for all } m_{1} \in W\right\}
$$

Proposition 2.4 Suppose $\chi(-1)=-1$. Then $W$ is self dual under $[\quad, \quad]$.

Proof: By Lemma 2.6

$$
\Phi\left(\tau\left(m_{1}\right), m_{2}\right)=-\Phi\left(\tau\left(m_{2}\right), m_{1}\right)
$$

for all $m_{1}, m_{2} \in M$. So for all $m_{1}, m_{2} \in W$ we have

$$
\Phi\left(m_{1}, m_{2}\right)=\Phi\left(\tau\left(m_{1}\right), m_{2}\right)=-\Phi\left(\tau\left(m_{2}\right), m_{1}\right)=-\Phi\left(m_{2}, m_{1}\right)=-\overline{\Phi\left(m_{1}, m_{2}\right)} .
$$

We know that $|M|=|W| \times\left|W^{\prime}\right|$ and $|W|=\sqrt{|M|}$, so $W=W^{\prime}$. This completes the proof.

Suppose $p=7$ and in Proposition 2.1 define $\chi: \mathbf{Z}_{7}^{*} \longrightarrow \mathbf{Z}_{4}[\omega]^{*}$ such that $\chi(5)=-\omega$ and choose $\zeta=1$ which is one of the solutions of (12). In this case by calculation, the space which is spanned by the rows of the following matrix over $\mathbf{Z}_{4}$ has rank 8 over $\mathbf{Z}_{4}$. Therefore $W$ is spanned over $\mathbf{Z}_{4}$ by the rows of this matrix.

$$
\left[\begin{array}{cccccccc}
\omega & \omega^{2} & \omega^{2} & \omega^{2} & \omega^{2} & \omega^{2} & \omega^{2} & \omega^{2} \\
-\omega^{2} & \omega & -\omega^{2} & -\omega & 1 & -1 & \omega & \omega^{2} \\
-\omega^{2} & \omega^{2} & \omega & -\omega^{2} & -\omega & 1 & -1 & \omega \\
-\omega^{2} & 1 & \omega^{2} & \omega & -\omega^{2} & -\omega & 1 & -1 \\
-\omega^{2} & -1 & 1 & \omega^{2} & \omega & -\omega^{2} & -\omega & 1 \\
-\omega^{2} & 1 & -1 & 1 & \omega^{2} & \omega & -\omega^{2} & -1 \\
-\omega^{2} & -\omega & 1 & -1 & 1 & \omega^{2} & \omega & -\omega^{2} \\
-\omega^{2} & -\omega^{2} & -\omega & 1 & -1 & 1 & \omega^{2} & \omega
\end{array}\right]
$$

This $W$ is a sextic residue code. This construction generalizes that of Chapman[3].

The symmetrized weight enumerator of a code $W$ over $\mathbf{Z}_{4}[\omega]$ is defined as follows.

Consider a specific codeword $r \in W$. Now, let $n_{0}(r)$ be the number of zeroes in the codeword, $n_{1}(r)$ be the number of elements of $\mathbf{Z}_{4}[\omega]^{*}, n_{2}(r)$ be the number of elements of $2 \mathbf{Z}_{4}[\omega]-\{0\}$ in the codeword. The symmetrized weight enumerator of $W$ is

$$
s w e_{W}(x, y, z)=\sum_{r \in W} x^{n_{0}(r)} y^{n_{1}(r)} z^{n_{2}(r)} .
$$


The symmetrized weight enumerator of $W$, the sextic residue code of length 8 is as follows.

$$
\begin{aligned}
\text { swe }_{W}(x, y, z)=x^{8} & +42 x^{4} z^{4}+672 x^{3} y^{4} z+2688 x^{2} y^{6}+2016 x^{2} y^{4} z^{2} \\
& +168 x^{2} z^{6}+16128 x y^{6} z+4704 x y^{4} z^{3}+11520 y^{8} \\
& +24192 y^{6} z^{2}+3360 y^{4} z^{4}+45 z^{8}
\end{aligned}
$$

\section{The Leech lattice}

Now we are going to construct the Leech lattice and one of the Niemeier lattices by using a higher power residue code of length 8 over $\mathbf{Z}_{4}[\omega]$.

We are going to use the same action of $\mathbf{S L}(2,7)$ on the code. Under this action for each $A=\left(\begin{array}{ll}\alpha & \beta \\ \gamma & \delta\end{array}\right) \in \mathbf{S L}(2,7)$

$$
e_{v} A=\vartheta(A, v) e_{w}
$$

where $w=\frac{\alpha v+\gamma}{\beta v+\delta}$ and $\vartheta(A, v)=\sigma(A, v) \omega^{j(A, v)}$, where $\sigma(A, v)$ is either 1 or -1 and $j(A, v)$ is 0,1 or -1 . We regard $j(A, v)$ are lying in the integers modulo 3 . It is also apparent that for each $A \in \mathbf{S L}(2,7)$ there exists an invertible $8 \times 8$ matrix $\hat{A}$ such that

$$
e_{v} A=e_{v} \hat{A}
$$

Lemma 3.1 Let $\vartheta(A, v)$ be defined by (20). Then

$$
\vartheta(A B, v)=\vartheta(A, v) \vartheta(B, v \cdot A)
$$

\section{Proof:}

$$
\begin{aligned}
\left(e_{v} A\right) B & =\vartheta(A, v) e_{v \cdot A} B \\
& =\vartheta(A, v) \vartheta(B, v \cdot A) e_{(v \cdot A) \cdot B} .
\end{aligned}
$$

On the other hand,

$$
e_{v}(A B)=\vartheta(A B, v) e_{v \cdot A B}
$$

Since the left hand sides are equal, the proof is complete.

Let $W$ be the sextic residue code of length 8 over $\mathbf{Z}_{4}[\omega]$ with character $\chi$ where $\chi(-1)=-1$ and $\chi(5)=-\omega$. Each $\zeta \in \mathbf{Z}_{4}[\omega]$ can be written uniquely as $\zeta=a_{0}+a_{1} \omega+a_{2} \omega^{2}, a_{i} \in \mathbf{Z}_{4}$ where $a_{0}+a_{1}+a_{2}=0$. 
Let $\hat{M}$ be a free $\mathbf{Z}_{4}$-module generated by $\left\{f_{\alpha, j}: \alpha \in \mathbf{P}^{1}\left(\mathbf{Z}_{7}\right), 0 \leq j \leq 2\right\}$. So we define

$$
\begin{array}{rlr}
\hat{\phi}: M & \rightarrow & \hat{M} \\
\zeta e_{\alpha} & \mapsto a_{0} f_{\alpha, 0}+a_{1} f_{\alpha, 1}+a_{2} f_{\alpha, 2}
\end{array}
$$

The map (22) can be easily extended to the map $\phi: W \rightarrow \hat{M}$ which takes $r \in W$ to

$$
\left(a_{\infty, 0}, a_{\infty, 1}, a_{\infty, 2}, a_{0,0}, a_{0,1}, a_{0,2}, \ldots, a_{6,0}, a_{6,1}, a_{6,2}\right)
$$

where

$$
a_{\alpha, 0}+a_{\alpha, 1}+a_{\alpha, 2} \equiv 0(\bmod 4) \quad \text { for } \alpha \in \mathbf{P}^{1}\left(\mathbf{Z}_{7}\right)
$$

We denote the code $\phi(W)$ by $T$.

We consider the matrix (18) and we replace each array by its three coordinates as above. So we have a generator matrix for the code $T$ over $\mathbf{Z}_{4}$ as follows.

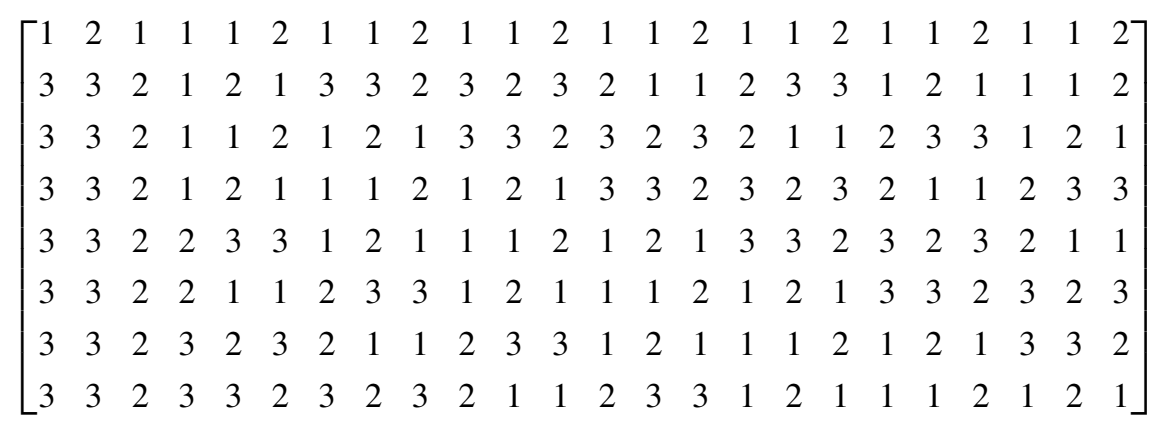

We consider the inner product on $\hat{M}$ with respect to the inner product with the $f_{\alpha, i}$ orthonormal and we set the weight of 0, 1, 2, 3 in $\mathbf{Z}_{4}$ as $0,1,4,1$ respectively. So the Euclidean weight of a codeword $r$ is the sum of the weights of its coordinates. It can be easily seen that the Euclidean weight of each codeword in $T$ is divisible by 8 . This shows that the code $T$ is self-orthogonal.

We shall define an action of $\mathbf{S L}(2,7)$ on $\hat{M}$. Define,

$$
f_{v, i} A=\sigma(A, v) f_{v \cdot A, i+j(A, v)}
$$

where the suffix $i+j(A, v)$ read modulo 3 . We can show that this action is 
well-defined.

$$
\left(f_{v, i} A\right) B=\sigma(A, v) f_{v \cdot A, i+j(A, v)} B=\sigma(A, v) \sigma(B, v \cdot A) f_{(v \cdot A) \cdot B, i+j(A, v)+j(B, v \cdot A)}
$$

On the other hand,

$$
f_{v, i} A B=\sigma(A B, v) f_{v \cdot A B, i+j(A B, v)}
$$

and by Lemma $3.1 \sigma(A, v) \sigma(B, v \cdot A)=\sigma(A B, v)$ and $j(A, v)+j(B, v \cdot A)=j(A B, v)$ which completes the proof.

Now it is easy to see that $\phi$ is $\mathbf{Z}_{4}$-linear and $\phi(r A)=\phi(r) A$ for all $r \in W$ and $A \in$ $\mathbf{S L}(2,7)$.

Proposition 3.1 Suppose $\bar{W}$ is a code of length 8 over $\mathbf{Z}_{4}[\omega]$ with generator matrix $\bar{G}$ and $\hat{A}$ is the matrix which is defined by (21). If $\bar{G} \hat{A}=\hat{A} \bar{G}$ then $\bar{W}$ is invariant under the action of $\mathbf{S L}(2,7)$.

Proof: Let $\bar{\zeta} \in \bar{W}$. Therefore, there exists $\bar{a} \in \mathbf{Z}_{4}^{8}$ such that $\bar{\zeta}=\bar{a} \bar{G}$, hence

$$
\bar{\zeta} \hat{A}=\bar{a} \bar{G} \hat{A}=\bar{\zeta} \hat{A} \bar{G}=\bar{\eta} \bar{G} \in \bar{W},
$$

for some $\bar{\eta} \in \mathbf{Z}_{4}^{8}$. This completes the proof.

Now consider the construction of the extended quaternary quadratic residue codes. Let $H$ be the matrix defined by $(10)$ with $\chi(\alpha)=\left(\frac{\alpha}{7}\right)$. Set $\widetilde{G}=5 I_{8 \times 8}-Y$. The matrix $H$ is a skew symmetric matrix and $\hat{A} \widetilde{G}=\widetilde{G} \hat{A}$, so by Proposition 3.1, $\widetilde{G}$ generates a code over $\mathbf{Z}_{4}$ which is invariant under the action of $\mathbf{S L}(2,7)$. Suppose $\operatorname{row}(G, i)$ is the $i$ th row of the matrix $G$. Now set

$$
\Theta=\left\{\frac{1}{2}( \pm \operatorname{row}(\widetilde{G}, i) \pm \operatorname{row}(\widetilde{G}, j)): 1 \leq i, j \leq 8\right\} .
$$

The code $Q_{4}$ which is generated by $\Theta$ is the extended quadratic residue code over $\mathbf{Z}_{4}$ obtained by Hensel lifting (see [4]). The code $Q_{4}$ is invariant under the action of $\mathbf{S L}(2,7)$ (see [2]). The number of linearly independent vectors in this set is at most 8 . So suffices it to consider 8 vectors as follows. Set the matrix $\hat{G}$ as a matrix where

$$
\operatorname{row}(\hat{G}, i)=\operatorname{row}(\widetilde{G}, 1)+\operatorname{row}(\widetilde{G}, i)) / 2
$$


That is

$$
\hat{G}=\left[\begin{array}{cccccccc}
1 & -1 & -1 & -1 & -1 & -1 & -1 & -1 \\
-1 & 2 & 0 & 0 & -1 & 0 & -1 & -1 \\
-1 & -1 & 2 & 0 & 0 & -1 & 0 & -1 \\
-1 & -1 & -1 & 2 & 0 & 0 & -1 & 0 \\
-1 & 0 & -1 & -1 & 2 & 0 & 0 & -1 \\
-1 & -1 & 0 & -1 & -1 & 2 & 0 & 0 \\
-1 & 0 & -1 & 0 & -1 & -1 & 2 & 0 \\
-1 & 0 & 0 & -1 & 0 & -1 & -1 & 2
\end{array}\right] .
$$

The code over $\mathbf{Z}_{4}$ which is spanned by the rows of the matrix $\hat{G}$ is the same as the code generated by

$$
G=\left[\begin{array}{cccccccc}
-1 & 1 & 2 & 1 & -1 & 0 & 0 & 0 \\
-1 & 0 & 1 & 2 & 1 & -1 & 0 & 0 \\
-1 & 0 & 0 & 1 & 2 & 1 & -1 & 0 \\
-1 & 0 & 0 & 0 & 1 & 2 & 1 & -1
\end{array}\right]
$$

which is the extended quadratic residue code over $\mathbf{Z}_{4}$ obtained by Hensel lifting [10, Chapter 11].

Define

$$
\begin{aligned}
\psi: \mathbf{Z}_{4}^{8} & \rightarrow \mathbf{Z}_{4}^{24} \\
\sum_{\alpha} a_{\alpha} e_{\alpha} & \mapsto \sum_{\alpha} \sum_{j=0}^{2} a_{\alpha} f_{\alpha, j} .
\end{aligned}
$$

Since $Q_{4}$ is a self-dual code then $Q^{(4)}=\psi\left(Q_{4}\right)$ is self-orthogonal. One can easily see that $Q^{(4)}$ is orthogonal to $T$. Moreover, $Q^{(4)} \cap T=0$. So the code $Q^{(4)}+T$ is a self-orthogonal of dimension 12. So it is self-dual. We denote the code $Q^{(4)}+T$ by $\Gamma$. A code over $\mathbf{Z}_{4}$ which is self-dual and the Euclidean weight of each codeword is divisible by 8 is called a code of type II. Computer calculation shows that the symmetric weight enumerator of $\Gamma$ is

$$
\begin{aligned}
\text { swe }_{\Gamma}=x^{24} & +759 x^{16} z^{8}+12144 x^{14} y^{8} z^{2}+170016 x^{12} y^{8} z^{4}+2576 x^{12} z^{12} \\
& +61824 x^{11} y^{12} z+765072 x^{10} y^{8} z^{6}+1133440 x^{9} y^{12} z^{3}+24288 x^{8} y^{16} \\
& +1214400 x^{8} y^{8} z^{8}+759 x^{8} z^{16}+4080384 x^{7} y^{12} z^{5}+680064 x^{6} y^{16} z^{2} \\
& +765072 x^{6} y^{8} z^{10}+4080384 x^{5} y^{12} z^{7}+1700160 x^{4} y^{16} z^{4} \\
& +170016 x^{4} y^{8} z^{12}+1133440 x^{3} y^{12} z^{9}+680064 x^{2} y^{16} z^{6} \\
& +12144 x^{2} y^{8} z^{14}+61824 x y^{12} z^{11}+4096 y^{24}+24288 y^{16} z^{8}+z^{24} .
\end{aligned}
$$


Now we consider the lattice in $\mathbf{R}^{24}$ associated with $\Gamma$ which is

$$
L_{\Gamma}=\left\{\frac{1}{2}(g+4 z): g \in \Gamma, z \in \mathbf{Z}^{24}\right\},
$$

where $g$ is regarded as $n$-tuples with integers $0,1,2,3$ as components. This construction is called construction $A_{4}$. Since the code $\Gamma$ is of type II then the lattice $L_{\Gamma}$ is an even unimodular lattice. As we see the number of the vectors of norm 2 is zero, $L_{\Gamma}$ is isomorphic to the Leech lattice ([6] chapter 18).

Bonnecaze et al. [2] have constructed the Leech lattice by using a different code over $\mathbf{Z}_{4}$, but they have found the same symmetrized weight enumerator. We show that these codes are not isomorphic.

Let $\bar{Q}$ be the code described in [2]. We show that $7 \nmid|A u t(\bar{Q})|$. Since we have shown that the automorphism group of $\Gamma$ contains $\mathbf{S L}(2,7)$, the conclusion would be apparent.

Theorem 3.1 The code $\Gamma$ is inequivalent to the code $\bar{Q}$.

Proof: The code is actually a lifting of the binary Golay code. Define

$$
\rho: \operatorname{Aut}(\bar{Q}) \rightarrow \operatorname{Aut}(\mathcal{G})
$$

where $\mathcal{G}$ is the Golay code and the image of an element is the element modulo 2. The image of $\rho$ is a group of automorphisms of the Golay code and $\operatorname{Aut}(\bar{Q}) \supseteq \operatorname{SL}(2,23)$ but $\rho(\mathbf{S L}(2,23))=\mathbf{P S L}(2,23)$, so PSL $(2,23) \subseteq \operatorname{Im} \rho$. We know that $M_{24}$ is the full automorphism group of the Golay code $\mathcal{G}_{24}$. So we have

$$
\operatorname{PSL}(2,23) \subseteq \operatorname{Im} \rho \subseteq M_{24}
$$

But PSL(2, 23) is maximal in $M_{24}$ [7], hence either $\operatorname{Im} \rho=\operatorname{PSL}(2,23)$ or $\operatorname{Im} \rho=M_{24}$. We show that $\operatorname{Im} \rho \neq M_{24}$. Suppose $\operatorname{Im} \rho=M_{24}$. Now consider a word $\varpi$ of shape $\left(\begin{array}{lll} \pm 1)^{8} & 2^{2} & 0^{14}\end{array}\right)$ in $\bar{Q}$. Let $O$ be an 8 element set (octad) formed by the positions of the $\pm 1 \mathrm{~s}$ in the word $\varpi$. The stabilizer of an octad is one of the maximal subgroups of $M_{24}$ and it acts 2-transitively on the remaining points. That means for $i, j, k, l$ which are not in the $O$ and $i \neq j, k \neq l$, we can find $g$ in the stabilizer of $O$ such that $g(i, j)=(k, l)$. There are 759 octads and by acting on $\pm \varpi$ by the octad stabilizer we get at least $2\left(\begin{array}{c}16 \\ 2\end{array}\right)$ words of shape $\left(( \pm 1)^{8} 2^{2} 0^{14}\right)$ in $\bar{Q}$ with \pm 1 s forming the octad $O$. So in total there are at least $2 \times 759 \times\left(\begin{array}{c}16 \\ 2\end{array}\right)=759 \times 16 \times 15$ elements of shape $\left(( \pm 1)^{8} 2^{2} 0^{14}\right)$ in $\bar{Q}$. But it is not possible due to $s w e_{\Gamma}$. Therefore, $\operatorname{PSL}(2,23)=\operatorname{Im} \rho$.

Any element of $\operatorname{ker} \rho$ is a diagonal matrix with \pm 1 s on diagonal, so has order 1 or 2 . Since $\operatorname{ker} \rho$ is a 2-group then 7 does not divide the $|A u t(\bar{Q})|$. This completes the proof. (I am indebted to Robin Chapman for this argument).

Now suppose $\lambda$ is a unit of $\mathbf{Z}_{4}[\omega]$. We know that $W$ is a $\mathbf{Z}_{4}$-linear code but not a $\mathbf{Z}_{4}[\omega]-$ linear code. Moreover, $\tau(\lambda r)=\bar{\lambda} \tau(r)$ for each $r \in W$ and $\bar{\lambda} \tau(r)=\lambda r$ if and only if $\lambda=\bar{\lambda}$. Therefore if $\lambda \in \mathbf{Z}_{4}[\omega]^{*}$ and $\lambda \bar{\lambda} \neq 1, \hat{W}=\lambda W$ is a different code from $W$ but 
$s w e_{W}=s w e_{\hat{W}}$. Replacing $W$ by $\lambda W$ in the above construction of $\Gamma$ gives a type II code $\hat{\Gamma}$. By applying the same process which is described in Section 3 we will find a different lattice. In fact, computer calculation shows that $s w e_{\hat{\Gamma}}$ is as follows

$$
\begin{aligned}
\text { swe }_{\hat{\Gamma}}=x^{24} & +48 x^{16} y^{8}+759 x^{16} z^{8}+11760 x^{14} y^{8} z^{2}+171360 x^{12} y^{8} z^{4} \\
& +2576 x^{12} z^{12}+61824 x^{11} y^{12} z+762384 x^{10} y^{8} z^{6}+1133440 x^{9} y^{12} z^{3} \\
& +24288 x^{8} y^{16}+1217760 x^{8} y^{8} z^{8}+759 x^{8} z^{16}+4080384 x^{7} y^{12} z^{5} \\
& +680064 x^{6} y^{16} z^{2}+762384 x^{6} y^{8} z^{10}+4080384 x^{5} y^{12} z^{7} \\
& +1700160 x^{4} y^{16} z^{4}+171360 x^{4} y^{8} z^{12}+1133440 x^{3} y^{12} z^{9} \\
& +680064 x^{2} y^{16} z^{6}+11760 x^{2} y^{8} z^{14}+61824 x y^{12} z^{11}+4096 y^{24} \\
& +24288 y^{16} z^{8}+48 y^{8} z^{16}+z^{24} .
\end{aligned}
$$

As we see the number of the words with minimum weight is 48 . If $L \subset \mathbf{R}^{n}$ is a root lattice and $R$ is its set of roots then the number $h=\frac{|R|}{n}$ is called the Coxeter number. Therefore the Coxeter number in this case is 2 and this lattice is equivalent to the Niemeier lattice $A_{1}^{24}$. See [6, Chapter 16] for the classification of Niemeier lattices.

\section{References}

1. W. A. Adkins and S.H. Weintraub, Algebra, Springer Verlag, New York, 1992.

2. A. Bonnecaze, P. Solé, and A.R. Calderbank, "Quaternary quadratic residue codes and unimodular lattices," IEEE Trans. Inform. theory 41(2) (1995).

3. R. Chapman, "Higher power residue codes," Finite Fields Appl. 3 (1997), 353-369.

4. R. Chapman, "Conference matrices and unimodular lattices," European J. Combin. 22 (2001), 1033-1045.

5. R. Chapman, Personal communication.

6. J. H. Conway and N. J. A. Sloane, Sphere Packings, Lattices and Groups, 1st edn., Springer Verlag, New York, 1988.

7. R. T. Curtis, “The maximal subgroups of $M_{24}$," Math. Proc. Cambridge Philos. Soc. 81 (1977), 185-192.

8. F. J. MacWilliams and N. J. A. Sloane, The Theory of Error-Correcting Codes, Elsevier Science Publishers, Amsterdam, 1991.

9. J. H. van Lint and R. M. Wilson, A Course in Combinatorics, Cambridge University Press, New York, 1992.

10. Zhe-Xian Wan, Quaternary Codes, World Scientific Publishing, London, 1997. 